\title{
Probabilistic Value-Centric Optimization Design for Fractionated Spacecrafts Based on Unscented Transformation
}

\author{
Ming Xu, ${ }^{1}$ Jinlong Wang, ${ }^{1}$ Ang Zhang, ${ }^{1}$ and Shengli Liu ${ }^{2}$ \\ ${ }^{1}$ Department of Aerospace Engineering, School of Astronautics, Beihang University, Beijing 100191, China \\ ${ }^{2}$ DFH Satellite Co., Ltd., Beijing 100094, China \\ Correspondence should be addressed to Ming Xu; xuming@buaa.edu.cn
}

Received 30 January 2013; Revised 19 March 2013; Accepted 8 April 2013

Academic Editor: Suiyang Khoo

Copyright (c) 2013 Ming Xu et al. This is an open access article distributed under the Creative Commons Attribution License, which permits unrestricted use, distribution, and reproduction in any medium, provided the original work is properly cited.

\begin{abstract}
Fractionated spacecrafts are of particular interest for pointing-intensive missions because of their ability to decouple physically the satellite bus and some imaging payloads, which possess a lesser lifecycle cost than a comparable monolithic spacecraft. Considering the probabilistic uncertainties during the mission lifecycle, the cost assessment or architecture optimization is essentially a stochastic problem. Thus, this research seeks to quantitatively assess different spacecraft architecture strategies for remote-sensing missions. A dynamical lifecycle simulation and parametric models are developed to evaluate the lifecycle costs, while the mass, propellant usage, and some other constraints on spacecraft are assessed using nonparametric, physics-based computer models. Compared with the traditional Monte Carlo simulation to produce uncertain distributions during the lifecycle, the unscented transformation is employed to reduce the computational overhead, just as it does in improving the extended Kalman filter. Furthermore, the genetic algorithm is applied to optimize the fractionated architecture based on the probabilistic value-centric assessments developed in this paper.
\end{abstract}

\section{Introduction}

The Defense Advanced Research Projects Agency (DARPA) implemented the Future, Fast, Flexible, Fractionated, FreeFlying (F6) program, aiming to demonstrate the concept of the fractionated architecture [1]. DARPA conducted extensive researches for the technological performances and economic advantages and invested Lockheed Martin Company (LM), Northrop Grumman Corporation (NG), Orbital Sciences Corporation (OSC), and Boeing Company (BC) for fractionated spacecrafts based on value-centric design methodologies (VCDM) in first phase of the F6 program [2]. This innovative design method for spacecraft has drawn great attentions from the astronautical community.

Considering the application of fractionated modules into responsive space, Richards et al. [3] analyzed the internal relations between the four aspects of technological, organizational, economic, and political supports. Brown and Eremenko $[4,5]$ summarized the achievements of VCDM in the F6 program from the viewpoint of the relationship between the innovative value-centered design standard and traditional monolithic design standard and then evaluated the concept of the fractionated spacecrafts compared with the traditional spacecraft architecture [5]. Mathieu and Weigel [6] evaluated the advantages and costs of the fractionated architecture in the fields of the attributes, strategies, and models. O'Neill [7] developed the semianalytic tool PIVOT for the model frame, risk, and the net present value and then optimized the PIVOT tool involved in the second phase of the F6 program. O'Neil and Mankins [8] achieved the conclusion that the fractionated spacecrafts were better on the lifecycle cost than a traditional spacecraft through the dynamical simulation and parameter models on the quality of the spacecraft [9]. Lafleur and Saleh $[10,11]$ developed another design tool GTFAST, which instanced specific analysis on the F6 program for handling input, model, and attributes. Yao et al. [12, 13] proposed the multidisciplinary optimization about the uncertainty for the spacecraft conceptual design and verified the feasibility and effectiveness of this optimization. Daniels et al. [14] presented a heuristics-based decision model using 
a Monte Carlo simulation to produce value distributions for satellite operator decision sets and a multi-stage decision process utilizing a dynamic programming algorithm to find value optimal decisions. Compared to the traditional measure metric on spacecraft cost, Collopy [15] derived a new metric rigorously from the view of probability, which places a focus on improving the probability of success rather than on reducing the cost.

One element necessary in enabling a probabilistic, valuecentric analysis of such fractionated architecture is a systematic method for sizing and costing many candidate architectures that arise in aerospace engineering. In this paper, a dynamical lifecycle simulation and parametric models are developed to evaluate the lifecycle costs, while the mass, propellant usage, and some other constraints on spacecraft are assessed using nonparametric, physics-based computer models. The lifecycle is divided into three phases, that is, module development, launching, and on-orbit control. Furthermore, the genetic algorithm is applied to optimize the fractionated architecture based on the probabilistic valuecentric assessments developed in this paper. To accelerate the optimization, one of the new techniques is to employ the graphic processing unit (GPU) accelerated genetic algorithm based on the Compute Unified Device Architecture (CUDA) to improve the searching efficiency because the assessments in any generation are parallel computerized. Another is to introduce the unscented transformation to reduce the amount of computations rather than the Monte Carlo simulation, just as it does in improving the extended Kalman filter (EKF) [16, 17].

\section{Cost, Value, and Assessment Indicators}

The cost assessment for a spacecraft design scheme includes module development costs, launch and operation costs, and risk costs from uncertainties during the lifecycle. All the types of the above costs are quantified by the monetary unit, for example, US dollar. The statistical sum of all the costs from the preparation phase to the end of the lifecycle constitutes the total cost of the spacecraft design scheme.

There are several valuation standards in the value-centric design methodologies to measure the practical spacecraft scheme by the quantitative assessments of the cost and value of fractionated spacecrafts. Quantitative assessment of the value gives a measurement indicator, as well as an evaluation methodology to provide information for the task of decision making. Considering the probabilistic uncertainties during the mission lifecycle, the value is acquired at the end of the lifecycle of the spacecraft from the quantitative criteria to quantify the value of spacecraft.

To study the cost and value of the trade-offs, the net present value (NPV) is usually used in the financial evaluation of the business assets $[10,11]$. It refers to the investment scheme generating net cash flow to the capital cost for the discount rate discount and original investment present value variance. Therefore, the net present value NPV is used to judge the fractionated spacecraft design in this paper. The higher the value of NPV is, the better the spacecraft design scheme is, which means that the input cost is low but the
TABLE 1: Parameter settings for fractionated components.

\begin{tabular}{lccccc}
\hline $\begin{array}{l}\text { Components } \\
\text { names }\end{array}$ & Weight $(\mathrm{kg})$ & Power $(\mathrm{W})$ & Cost $(\mathrm{M} \$)$ & TRL & FIT \\
\hline EO & 40 & 15 & 15 & 9 & 5000 \\
24/7 Comm & 4 & 25 & 5 & 5 & 5000 \\
HBD & 10 & 25 & 2 & 9 & 5000 \\
SSR & 8 & 100 & 2 & 7 & 6000 \\
MDP & 8 & 18 & 1 & 6 & 5000 \\
AIS & 5 & 15 & 0.5 & 8 & 3500 \\
\hline
\end{tabular}

output value is high. Hence, the input-output ratio could be weighted by the NPV value, which is formulized as follows [5]:

$$
\mathrm{NPV}=\frac{N}{\left(1+D_{R}\right)^{T_{\text {yearloop }}-1}},
$$

where $D_{R}$ is the discount rate, $N$ is the free cash flow, and $T_{\text {yearloop }}$ is the spacecraft lifecycle (unit: year).

\section{Spacecraft Models}

3.1. Fractionated Spacecraft Architecture. The spacecraft architecture modeling is a prerequisite to assess the cost and value of the spacecraft design scheme. But the fractionated spacecrafts are quite different from traditional spacecraft because of the different design principles on the two types of architectures. Common architecture level definitions on the fractionated spacecraft architecture modeling are listed from low to high order, as "component," "module," and "cluster" [12].

Component: it is the smallest unit in the fractionated spacecraft architecture modeling. The four companies modeled the components in the first phase of the DARPA mission, mainly including the following components: (1) payload component: the valuable payloads are the feasible assessment to increase the total value of the fractionated spacecraft for the purpose of using the least cost to achieve the more value; (2) measurement and control component: it realizes the continuous measurement and control through the relay module in the architecture constellation; (3) mission data processing component: usually the on-board computer is used for processing complex data; (4) digital communication component: it is responsible for communication between the space and ground, as well as responsible for the data download and upload; (5) data storage component: it is storing large amounts of data and preparing for data transmission, that is, solid-state drive (SSR).

Module: it consists of several components to achieve independent and free flight on orbit. In addition to payload and other functional components, it includes other associated components of the power supply and thermal control.

Cluster: it is composed of multiple fractionated modules in formation flight to complete certain missions.

Using six separable components in this paper, the parameters are shown in Table 1 [18], where FIT represents the number of failures in 1000 hours and the characterization 
of component reliability parameters, the Earth observing payload (EO) is employed throughout this paper to meet the imaging missions, $24 / 7$ Comm is the digital component, HBD is the high-bandwidth downloading communication component, SSR is the solid hard drive component, MDP is the mission data processing component, and AIS is the automatic identification component.

The spacecraft scheme includes the design of spacecraft architecture and the selection of launch vehicles [19]. To inject fractionated spacecrafts into space, six alternative categories for the launch vehicle are provided in this paper, that is, Minotaur I, Athena I, Taurus 2210, Taurus 3110, Minotaur IV, and Athena II [1], which have different launch capacities and reliabilities, and different launch vehicle costs. Therefore, the launch vehicle should be selected based upon the aerospace mission and the fractionated architecture.

The combination of separated components, the cluster segmentations, and the selection of launch vehicle impact the assessed cost and value of the fractionated spacecrafts. As a result, the optimization of the cluster segmentation and its separated components should be taken to get a higher inputoutput ratio.

\subsection{Spacecraft Architecture Design Models}

3.2.1. Cost Models. There are three parts of the fractionated spacecraft's cost: module development cost, launch and operation cost, and risk cost, where commercial insurance for spacecraft is involved in launch and operation cost, and the ground equipment cost and software development cost are not accounted in this paper. Risk cost includes the cost caused by time delay during the module development and the failure due to launch vehicle and on-orbit maintenance, which are considered to be governed by uncertainties.

According to the recyclability, the cost can be divided into nonrecyclable and recyclable types. Inherited from this attribution, the separated components in the cluster would be divided into the recyclable and nonrecyclable ones. This classification is primarily proposed for some reusable equipment and design schemes for the specified mission. In this case, the second cost is the result from the combination of the first cost and the learning curve rate.

(1) Module Development Costs. Different evaluations arise from the development costs with different components, or different development cycles and technology readiness levels (TRL) for the same components. For any spacecraft architecture scheme, the recyclable and nonrecyclable costs are calculated, respectively. For the specified type of modules, the cost on the module manufactured at the first time is higher than others because of its low TRL; however, the cost on the module built at the second or subsequent time is lower due to the cost of the recyclable components.

Considering the batch production, the cost of the unit module is estimated as follows:

$$
\begin{gathered}
C_{\bmod _{i 1}}=C_{\bmod _{i 1}}^{\mathrm{NRE}}+C_{\bmod _{i 1}}^{\mathrm{REC}}, \\
C_{\bmod _{i Q}}=C_{\bmod _{i 1}}^{\mathrm{REC}} \cdot Q^{\left(\ln L_{R}\right) / \ln 2},
\end{gathered}
$$

where $C_{\text {mod }_{i 1}}$ is the cost of the first module of the $i$ th type module including nonrecyclable cost $C_{\bmod _{i 1}}^{\mathrm{NRE}}$ and recyclable cost $C_{\bmod _{i 1}}^{\mathrm{REC}}, C_{\bmod _{i Q}}$ is the cost of the Qth module of the $i$ th type module, and $L_{R}$ is the learning curve rate of the module development and production. The nonrecyclable cost and recyclable cost are calculated by the components and satellite platform, the cost calculation formula with reference to the cost of small satellite model (abbr. SSCM07), which was developed by Mahr [20] for the Aerospace Cooperation and has potential applications in estimating the manufacturing cost empirically [10, 11, 13, 21].

SSCM07 is good at estimating the production cost; however, it is powerless in estimating other types of costs (such as operation, risk, and inflation cost) and their dynamic and uncertain evolutions during the spacecraft's lifecycle. Thus, we employed SSCM07 to estimate the production costs for fractionated modules and then developed a probabilistic method to measure all the uncertain costs during the lifecycle dynamically.

Taking inflation into account, the cost of the unit module is estimated as follows:

$$
C_{\bmod _{i Q}}^{\text {inflated }}=C_{\bmod _{i Q}} \cdot\left(1+R_{\text {inflated }}\right)^{T_{\text {lateryear }},}
$$

where $R_{\text {inflated }}$ is the year inflation rate and $T_{\text {lateryear }}$ is expressed as module development time (unit: year). All module's development cost is as follows:

$$
C_{M}=\sum_{i=1}^{N_{\text {type }}}\left(\sum_{j=1}^{\substack{N_{\text {tyod }}^{\text {tyo }} \\ \text { inflated }}} C_{\bmod _{i j}}^{\text {infla }},\right.
$$

where $N_{\text {type }}$ is the number of the spacecraft module's type and $N_{\bmod _{i}}^{\text {type }}$ is the number of certain type module.

(2) Launching and Operational Costs. The selection of a launch vehicle scheme is determined according to the module properties. Therefore, the cost of the launch vehicle is estimated by the design scheme of the spacecraft architecture. The requirement from the spacecraft and the capacity of the launch vehicle, respectively, restrict the feasible combinations between them, so the number of selecting launch vehicles is less than the number of modules. Thus, the launch cost is listed as follows:

$$
C_{\text {launch }}=\sum_{i=1}^{N_{\text {launch }}} C_{\text {launch }}^{i} \text {, }
$$

where $N_{\text {launch }}$ is the total expected number of launch vehicles and $C_{\text {launch }}^{i}$ is the cost of the $i$ th vehicle. Owning to the huge cost of launch vehicles, it is necessary to reduce the number of launch vehicles and to load as many modules as possible in every vehicle.

When the fractionated spacecrafts are on orbit, operating each module will generate the operational cost. In case of the annual operational $\operatorname{cost} C_{i}^{\text {ops }}$ for each module being equal to 
$2 \mathrm{M} \$$, the total operational cost $C_{\mathrm{ops}}$ during the spacecraft's lifecycle is as follows:

$$
C_{\mathrm{ops}}=\sum_{i=1}^{N_{\mathrm{type}}}\left(\sum_{j=1}^{\substack{N_{\text {mpd }}^{\mathrm{type}} \\ \text { mops }}} C_{i}^{\mathrm{ops}} \cdot T_{i j}\right),
$$

where $T_{i j}$ is the total orbiting time of the $i$ th type module on the $j$ th year. Then the launch and operational cost of fractionated spacecrafts during the lifecycle is as follows:

$$
C_{\text {lo }}=C_{\text {launch }}+C_{\text {ops }} \text {. }
$$

(3) Risk Costs. The uncertainties, such as task delay, launching failure, or on-orbit failure, exist in all the phases of spacecraft development, launching, and on-orbit operation, which are required to be maintained with extra costs. The risks are measured by the risk cost $C_{\text {risk }}$ as the criterion of the robustness and reliability in spacecraft architecture design, which are allocated by launching failure and on-orbit failure in this paper.

Launching failure: this type of failure comes from the reliability of launch vehicles less than $100 \%$. If a launch vehicle happens to fail, all the modules carried by this vehicle need to be redeveloped and then launched. Hence, the risk cost of launching failure can be formulized as follows:

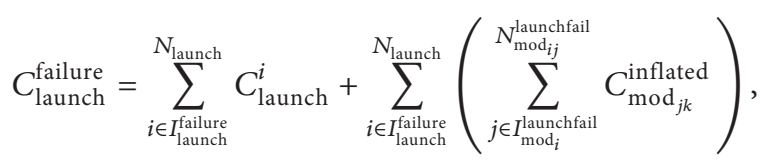

where $I_{\text {launch }}^{\text {failure }}$ is the identification number of the failing launch vehicle, $N_{\bmod _{i j}}^{\text {launchail }}$ and $I_{\bmod _{i}}^{\text {launchfail }}$ indicate the quantity and type of the modules carried by the failing vehicle " $i$," respectively.

On-orbit operation: if a module fails on-orbit, another module of the same type is expected to be launched to replace this faulty module. Thus, the cost of on-orbit operation is essentially the development and launching costs for the new module, which is listed as shown:

$$
C_{\mathrm{ops}}^{\text {failure }}=\sum_{i=1}^{N_{\text {ops }}^{\text {failure }}} C_{\text {launch }}^{i}+\sum_{i \in I_{\text {launch }^{\text {failure }}}}^{N_{\text {launch }}}\left(\sum_{j \in I_{\text {mod }_{i}}^{\text {opsfail }}}^{N_{\text {modij }}^{\text {opsfail }}} C_{\text {mod }_{j k}}^{\text {inflated }}\right),
$$

where $N_{\mathrm{ops}}^{\text {failure }}$ is the identification number of the module failing on-orbit, $N_{\bmod _{i j}}^{\text {opsaal }}$ and $i_{\bmod _{i}}^{\text {opsfail }}$ indicate the quantity and type of the module failing on-orbit, respectively.

Therefore, the risk cost can be considered as the failure cost during the launching or operational phase, as

$$
C_{\text {risk }}=C_{\text {launch }}^{\text {failure }}+C_{\text {ops }}^{\text {failure }} .
$$

According to the above sections presenting the costs when developing, launching, and operating a module and the risk costs due to the failures in these phases, the total cost of the fractionated architecture can be expressed as following:

$$
C=C_{M}+C_{\text {lo }}+C_{\text {risk }} \text {. }
$$

3.2.2. Value Models. The revenue model gives a finical rule measuring the spacecraft gains, which are considered as the data products achieved from the payload modules and their communication links. Consequently, some empirical weight factors are introduced to monetize the imaging data to model the benefit value of a spacecraft. When the data have better resolution and positioning accuracy, a larger factor is weighted to price them; on the other hand, a smaller weight factor is expected.

The effectiveness of the communication link affects the value from the imaging data as well, depending upon the type of link hardware (low or high speed transmission), the frequency, and duration of the spacecraft passing through the data receiving stations.

In this paper, the payloads are defined to employ both the low-rate downlink (abbr. LR), high-rate downlink (abbr. HR), and space-ground interlink (abbr. SG) to implement the digital transmissions. Thus, the benefit value of the fractionated spacecrafts can be derived from the following equation:

$$
R_{i+j}=\delta_{i}\left(N_{i}^{\mathrm{LR}}+N_{i}^{\mathrm{HR}}+N_{i}^{\mathrm{SG}}\right)+\delta_{j}\left(N_{j}^{\mathrm{LR}}+N_{j}^{\mathrm{HR}}+N_{j}^{\mathrm{SG}}\right),
$$

where $\delta_{i}$ and $\delta_{j}$ are the weight factors of the payloads, respectively, and $N_{k}^{\mathrm{LR}}, N_{k}^{\mathrm{HR}}$, and $N_{k}^{\mathrm{SG}}, k=i, j$, are, respectively, the amount of valid data obtained by the link between space and ground.

3.2.3. Uncertainty Models. A systematic study on the investing cost and economic benefit assessments on the spacecraft architectures is implemented in this paper. Considering the probabilistic uncertainties during the mission lifecycle, the cost assessment or architecture optimization is essentially a stochastic problem. The uncertainties such as financial inflation, launching failure, and on-orbit operating failure are required to be maintained by extra costs, which are formulized by (3) in Section 3.2.1(1) and (8), (9), and (10) in Section 3.2.1(3).

In the numerical assessments, all the uncertainties are modeled by the stochastic noises. Generally, the upper and lower bounds of these uncertainties are determined according to the experience in previous work or technology readiness level (TRL), which are formulized as following:

$$
C=F\left(C_{s}, \delta_{\max }, \delta_{\min }\right),
$$

where $C_{s}$ is the mean value and $\delta_{\max }$ and $\delta_{\min }$ are the upper and lower bounds, respectively. The annual inflation rate is assumed as the Gaussian noise approximately with its mean and variance assigned to $3.5 \%$ and $0.5 \%$, respectively, according to the historical consumer price index (CPI) of China. The reliability of launch vehicles is set as $P_{\text {launch }}=$ 95.1\% according to the handbook of the vehicle type of Minotaur IV, which means the launching failure occurs when a random number from the uniform distribution $U(0,1)$ is larger than the reliability $P_{\text {launch }}$. The on-orbit operating failure is dependent on the mean time to failure $\theta$ of the components, which is modeled by the exponential 
distribution as $f(t)=\lambda e^{-\lambda \cdot t}$, where $\lambda$ is referred as aging rate equal to $1 / \theta$ and is set as $5 \%$ in this paper.

For a specified design scheme, the traditional Monte Carlo simulation is employed by the cost and value assessment to cope with the uncertainties. More than 10000 scenarios are created by the Monte Carlo method to simulate all the combinations of the noises mentioned above. Generally, the Monte Carlo simulation costs a mass of computation to generate all the situations ergodicly. Therefore, this statistical method is feasible just for evaluating a specified design scheme within all kinds of scenarios; however, it is not available for refining the optimal one from many candidate schemes. For example, the best design scheme is expected to be selected from 5000 candidates, and then the optimization needs to be evaluated $5 \times 10^{7} \quad(=5000 \times 10000)$ times. The heavy computation makes the optimizing iteration very difficult or impossible.

Thus, it is necessary to introduce the unscented transformation (UT) firstly proposed by Julier and Uhlmann [16] to reduce the amount of computations rather than the Monte Carlo simulation. The most common use of UT is in the nonlinear projection of mean and covariance estimates in the context of nonlinear extensions of the Kalman filter [17]. The principal advantage of the approach is that the nonlinear function is fully exploited, as opposed to the extended Kalman filter which replaces it with a linear one. One immediate advantage is that the UT can be applied with any given function whereas linearization may not be possible for functions that are not differentiable. A practical advantage is that the UT can be easier to implement because it avoids the need to derive and implement a linearizing Jacobian matrix.

The mean and covariance of financial inflation, launching failure, and on-orbit operating failure will be exactly encoded by sigma points and then be propagated by the assessment procedure to each point. Hence, the mean and covariance of the transformed set of points then represent the desired transformed estimate.

The unscented transformation is defined as the application of a given function to any partial characterization of an otherwise unknown distribution, but its most common use is for the case in which only the mean and covariance are given. For the fractionated architecture with six modules, the random uncertainties (denoted by $\mathbf{x} \in \mathbf{R}^{6}$ ) from the annual inflation rate, launching failure, and on-orbit operating failure have the mean $\overline{\mathbf{x}}$ and variance $\mathbf{P}$. To yield the statistics of some combination of cost and value $y$ propagated through the nonlinear mapping defined by the cost and value assessment procedure $\mathbf{y}=\mathbf{h}(\mathbf{x})$, a set of 13 sigma points $\left\{\widetilde{\mathbf{x}}_{i}, i=0,1, \ldots, 12\right\}$ are calculated using the following general selection scheme [16]:

$$
\begin{gathered}
\mathbf{x}^{(0)}=\overline{\mathbf{x}}, \\
\widetilde{\mathbf{x}}^{(i)}=\overline{\mathbf{x}}+(\sqrt{6 \cdot \mathbf{P}})^{T}, \quad i=1,2, \ldots, 6, \\
\widetilde{\mathbf{x}}^{(i)}=\overline{\mathbf{x}}-(\sqrt{6 \cdot \mathbf{P}})^{T}, \quad i=7,8, \ldots, 12 .
\end{gathered}
$$

Once the sigma points are calculated from the prior statistics as shown above, they are propagated through the nonlinear

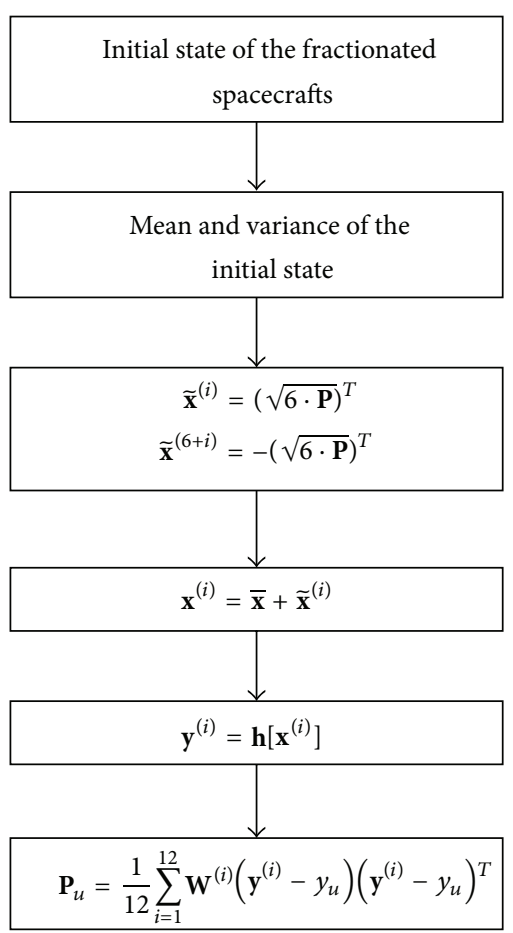

FIGURE 1: The process of applying UT to assess the cost and value for the fractionated architecture.

function $\mathbf{y}^{(i)}=\mathbf{h}\left[\mathbf{x}^{(i)}\right]$. Thus, the mean $y_{u}$ and variance $\mathbf{P}_{u}$ of $\mathbf{y}$ approximated using a weighted sample mean and covariance of the posterior sigma-points are as follows:

$$
\begin{gathered}
y_{u}=\frac{1}{12} \sum_{i=1}^{12} \mathbf{W}^{(i)} \mathbf{y}^{(i)}, \\
\mathbf{p}_{u}=\frac{1}{12} \sum_{i=1}^{12} \mathbf{W}^{(i)}\left(\mathbf{y}^{(i)}-y_{u}\right)\left(\mathbf{y}^{(i)}-y_{u}\right)^{T},
\end{gathered}
$$

where the weight coefficients $\mathbf{W}^{(i)}$ are restricted by the normalization condition $\sum_{i=0}^{12} \mathbf{W}^{(i)}=1$. The process of applying the unscented transformation to calculate the statistical result of the cost and value assessment for the fractionated architecture is presented in Figure 1.

Compared with the traditional Monte Carlo simulation, this approach characterizes a probability distribution only in terms of few set of statistics. Furthermore, considering the ideal case without the uncertainties, the cost and benefit value (including the net present value NPV) will be the same as the single result achieved from the initial state of the fractionated spacecrafts.

\section{Cost and Value Assessments}

4.1. Cost and Value Assessment Procedure. Cost and value assessment for the fractionated architecture is quite dependent on all the stages divided in the whole lifecycle, that is, module development, launching, and on-orbit operation. Within the cost and value accumulated in each stage, the total 


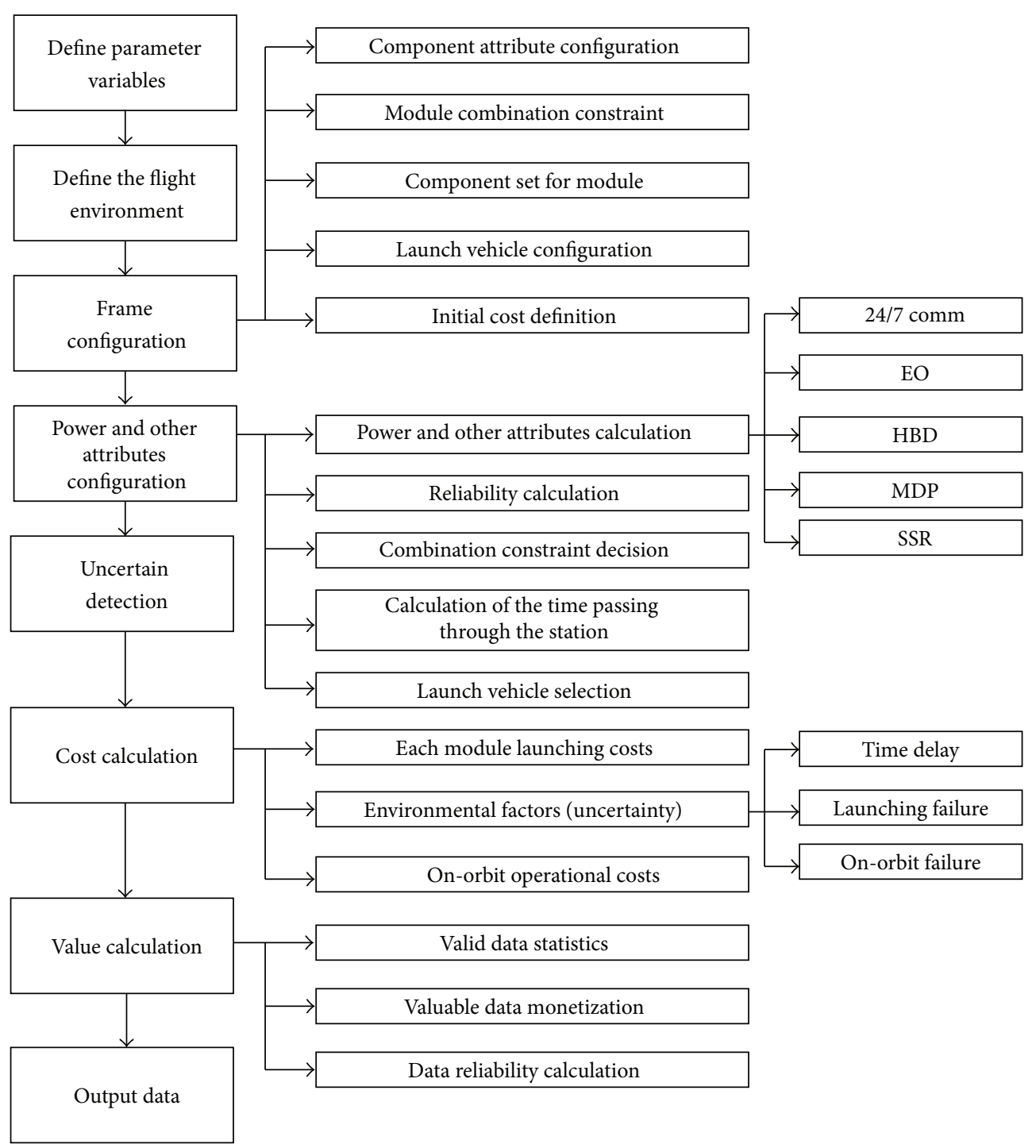

FIGURE 2: The flowchart of cost and value assessment for fractionated architecture.

cost and value will be yielded at the end of the lifecycle. The flowchart of the cost and value assessment is presented in Figure 2 for the fractionated architecture.

The main work is to define some relevant parameters for the fractionated spacecrafts in its early development stage, including setting the uncertainties due to the time delay and choosing the functional components assembled into the modules and their launch vehicles. The cost settlement at this stage should take the inflation rate and others into consideration. Moreover, no value is yielded in this stage because the spacecraft has not been injected into space and no imaging data are produced at the module development stage.

In the launch phase, the certain cost comes from a series of launch vehicles adapted by the developed modules, and the uncertain cost originates from the launching failure which requires more costs in redeveloping and relaunching modules. In addition, no value is yielded in this stage as well.

After injected into space successfully, the spacecraft is expected to be debugged on-orbit for about one month, and then some functional components (like on-board camera) will be put into use and able to produce valuable data, so that the spacecraft starts to benefit from this stage. Furthermore, the debugging stage costs the on-orbit operation a lot.

The uncertain cost in the operational stage results from a module failing on-orbit. In this case, another module is launched to replace this faulty module after redeveloped in accordance with the existing template. During the replacement, the faulty module and its successor have no contributions to the benefit value.

Therefore, the cost and value of the fractionated architecture can be obtained through the whole lifecycle according to the assessment flowchart listed in Figure 2.

4.2. Simulation of Cost and Value Assessment. In this paper, the nominal orbit is set as a sun-synchronous orbit with the orbital altitude of $500 \mathrm{~km}$ and the local time at descending node of 10:30 AM. The aerospace mission starts from 1st of January, and the development period of a module is assumed 
TABLE 2: The net present value NPV in five-year lifecycle.

\begin{tabular}{lc}
\hline Time & NPV \\
\hline The first year & -105.1077 \\
The second year & -144.0231 \\
The third year & 118.7044 \\
The fourth year & 132.8142 \\
The fifth year & 14.1471 \\
\hline
\end{tabular}

TABLE 3: The net present value NPV in ten-year lifecycle.

\begin{tabular}{lc}
\hline Time & NPV \\
\hline The first year & -95.1603 \\
The second year & -131.6379 \\
The third year & 55.4421 \\
The fourth year & 62.4822 \\
The fifth year & 51.2209 \\
The sixth year & 96.6323 \\
The seventh year & 62.1255 \\
The eighth year & 109.7646 \\
The ninth year & 119.1036 \\
The tenth years & 119.1036 \\
\hline
\end{tabular}

TABLE 4: Average cost and benefit (unit: M\$) in five-year lifecycle and their standard variances.

\begin{tabular}{lcccc}
\hline & $\begin{array}{c}\text { Average } \\
\text { cost }\end{array}$ & $\begin{array}{c}\text { Cost standard } \\
\text { variance }\end{array}$ & $\begin{array}{c}\text { Average } \\
\text { benefit }\end{array}$ & $\begin{array}{c}\text { Benefit standard } \\
\text { variance }\end{array}$ \\
\hline Value & 264.0081 & 32.1762 & 265.5474 & 59.9580 \\
\hline
\end{tabular}

TABLE 5: Average cost and benefit (unit: M\$) in ten-year lifecycle and their standard variances.

\begin{tabular}{ccccc}
\hline & $\begin{array}{c}\text { Average } \\
\text { cost }\end{array}$ & $\begin{array}{c}\text { Cost standard } \\
\text { variance }\end{array}$ & $\begin{array}{c}\text { Average } \\
\text { benefit }\end{array}$ & $\begin{array}{c}\text { Benefit standard } \\
\text { variance }\end{array}$ \\
\hline Value & 696.8454 & 37.8953 & 813.8701 & 88.1731 \\
\hline
\end{tabular}

as two years. All the modules are launched by the vehicle type of Minotaur IV with its reliability of $95.1 \%$. The simulation cases are implemented in this paper with the lifecycles of five and ten years, respectively.

Considering the cost and value uncertainties from schedule change, launching failure, on-orbit failure, commercial insurance, module replacement, and other factors, the net present values counted numerically in the lifecycles of five and ten years are shown in Tables 2 and 3, and the average costs and benefits are shown in Tables 4 and 5. Then the assessments in the lifecycles of five and ten years are classified and labeled on the phase plane of cost and benefit values shown in Figures 3 and 4, where the two error ellipses are yielded by the unscented transformation with the confidence levels of 0.5 and 0.68 , respectively, and the scattered points are plotted by the Monte Carlo method.

The numerical simulation indicates that the cost and benefit values quite depend on the mission lifecycle. The longer lifecycle increases the risk of on-orbit failure and the cost



FIGURE 3: The assessments in five-year lifecycle labeled on the phase plane of cost and benefit values.

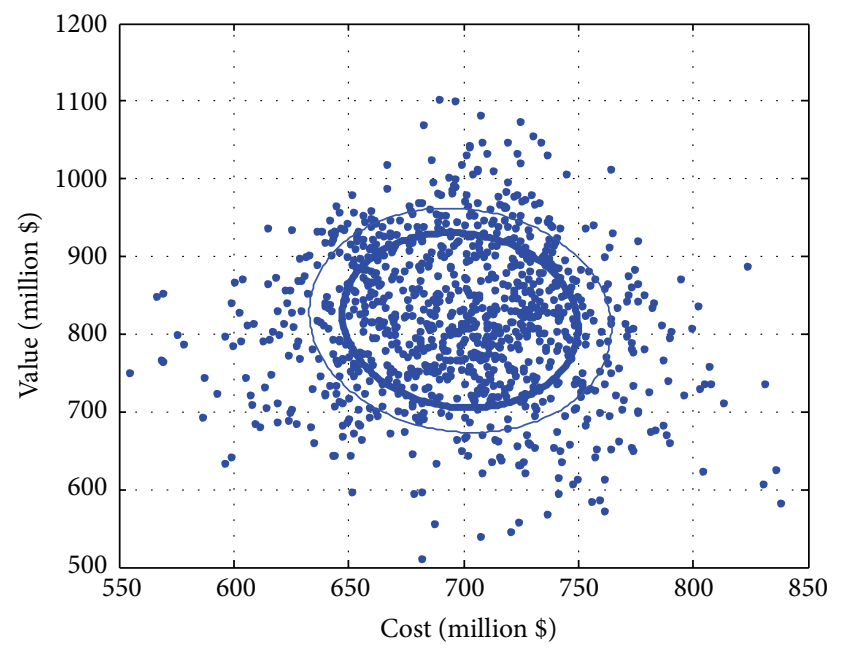

FIgURE 4: The assessments in ten-year lifecycle labeled on the phase plane of cost and benefit values.

of module replacement and produces more valuable imaging data which raises the benefit values, which accounts for why the cost and value created in the lifecycle of ten years are more than five years. Moreover, most of the scattered points located inside the error ellipses validate the feasibility of the unscented transformation. Therefore, the assessments of many candidate schemes will be implemented in Section 5 by the unscented transformation to accelerate the optimization procedure.

However, there exists no relationship between the lifecycle of the fractionated spacecrafts and the net present value, which is accumulated by all the uncertainties during the lifecycle. Furthermore, the points far away from the confidence ellipses may happen with a very low probability, so that they are often ignored in aerospace engineering. 

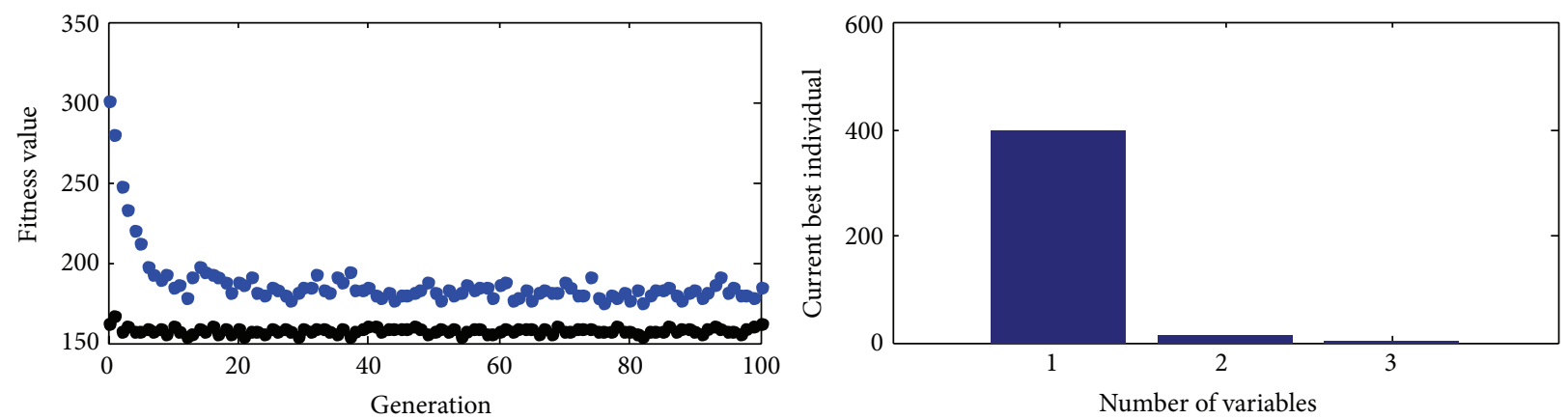

- Best fitness

- Mean fitness
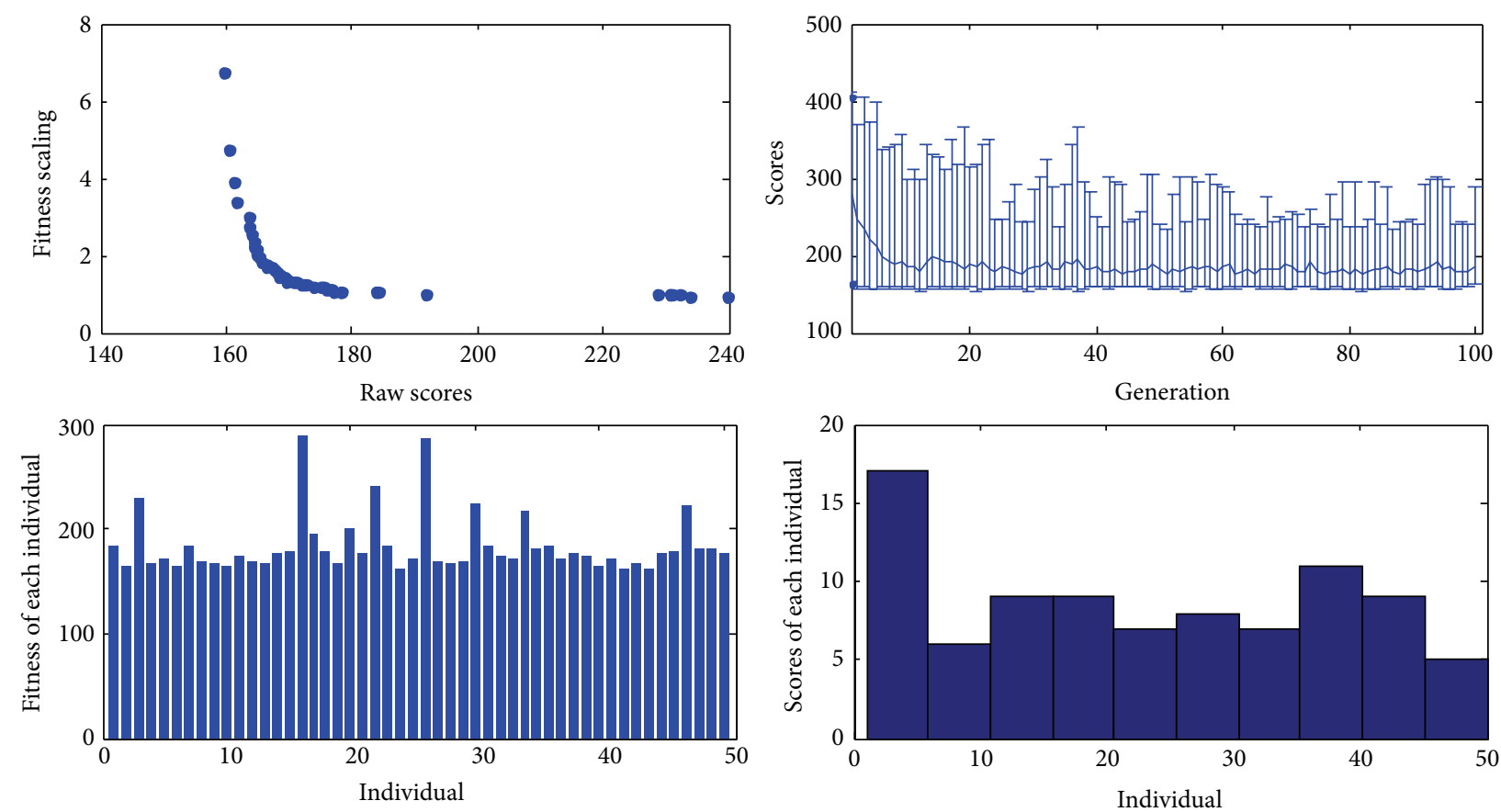

FIGURE 5: The optimization results by genetic algorithm.

\section{Probabilistic Value-Centric Optimization for Fractionated Spacecraft Architecture}

The value-centric assessment methodology can customize several criterions, such as the mean and variance of the cost, the benefit income, and the net present value NPV. Therefore, the optimization is essentially solving a multiobjective problem in Pareto's sense [13, 22].

For this multidisciplinary optimization problem, there exist analytic gradient functions to guide the refinement of iteration procedure of numerical optimization. Thus, the heuristic method, such as the genetic algorithm, is quite good at refining the optimal design scheme. Compared with the traditional algorithm, the graphic processing unit (GPU) accelerated genetic algorithm based on the Compute Unified Device Architecture (CUDA) is employed to improve the searching efficiency because the cost and value assessments in any generation are parallel computerized.
In this paper, the fractionated spacecrafts with fiveyears lifecycle are chosen to optimize their architecture. The objective function for this case is to maximize the net present value NPV with the optimized variables of orbital altitude, local time at descending node (LTDN), and the number of modules. The inequality constraints for the three variables are listed in Table 6.

For the genetic initialization, the population number within each generation is set as 100 . The evolution after the fifty generations can refine the maximum net present value NPV, shown in Figure 5. In the optimal design scheme, the orbital altitude is $573.7150 \mathrm{~km}$, the local time at descending node is 11:23 AM, and the number of modules employed by the fractionated architecture is six. Ideally, if the design models are accurate enough, the maximum NPV could be reached through the engineering practice.

According to the numerical simulations, the optimal design scheme of the fractionated architecture depends on 
TABLE 6: Inequality constraints for the three variables.

\begin{tabular}{lcc}
\hline Optimal conditions & Lower bound & Upper bound \\
\hline Orbit altitude $(\mathrm{km})$ & 400 & 700 \\
LTDN (hour) & 9 & 13 \\
Number of modules $(-)$ & 1 & 6 \\
\hline
\end{tabular}

the assessment criterions. For the high-budget missions requiring more reliability or robustness, the variance of cost, income, or net present value is preferable to working as the objective function; however, the mean is selected by some low-budget missions which pay more attentions on the costperformance ratio.

\section{Conclusion}

Considering the probabilistic uncertainties during the mission lifecycle, the cost assessment or architecture optimization is essentially a stochastic problem. One element necessary in enabling a probabilistic, value-centric analysis of such fractionated architecture is a systematic method for sizing and costing the many candidate architectures that arise. One of the contributions in this paper is to quantitatively assess the impacts of various fractionated spacecraft architecture strategies on the lifecycle cost, mass, propellant usage, and mission lifetime of pointing-intensive, remote-sensing mission spacecraft.

Based on the probabilistic value-centric assessments developed in this paper, the genetic algorithm is applied to optimize the fractionated spacecraft architecture from the viewpoint of probability. To accelerate the optimization, the second contribution is to employ the graphic processing unit (GPU) accelerated genetic algorithm based on the Compute Unified Device Architecture (CUDA) to improve the searching efficiency because the assessments in any generation are parallel computerized. Furthermore, another is to introduce the unscented transformation to reduce the amount of computations rather than the Monte Carlo method in stochastic simulation.

Finally, for future work, the models can ultimately be developed on a level of confidence such that the results of the surplus value model can be analyzed in detail, in particular to establish the design objective functions for components. This can then provide a platform for optimization to generate the best design scheme for different aerospace missions.

\section{Acknowledgments}

The research is supported by the National Natural Science Foundation of China (11172020), the National High Technology Research and Development Program of China (863 Program: 2012AA120601), Talent Foundation supported by the Fundamental Research Funds for the Central Universities, Aerospace Science and Technology Innovation Foundation of China Aerospace Science Corporation, and Innovation Fund of China Academy of Space Technology. The authors have no conflict of interests with the mentioned trademark(s).

\section{References}

[1] O. C. Brown, P. Eremenko, and P. D. Collopy, "Value-centric design methodologies for fractionated spacecraft: progress summary from phase 1 of the DARPA system F6 program," in Proceedings of the AIAA Space 2009 Conference and Exposition, AIAA 2009-6540, Pasadena, Calif, USA, September 2009.

[2] M. G. O’Neill, H. Yue, S. Nag, P. Grogan, and O. L. deWeck, "Comparing and optimizing the DARPA system F6 program value-centric design methodologies," in Proceedings of the AIAA Space 2010 Conference and Exposition, AIAA 2010-8828, Anaheim, Calif, USA, 2010.

[3] M. G. Richards, Z. Szajnfarber, M. G. O’Neill, and A. L. Weigel, "Implementation challenges for responsive space architectures," in Proceedings of the AIAA Responsive Space Conference, AIAARS7-2009-2004, Los Angeles, Calif, USA, April 2009.

[4] O. Brown and P. Eremenko, "Application of value-centric design to space architectures: the case of fractionated spacecraft," in Proceedings of the AIAA Space 2008 Conference and Exposition, AIAA 2009-6540, San Diego, Calif, USA, September 2008.

[5] O. Brown and P. Eremenko, "The value proposition for fractionated space architectures," in Proceedings of the Space 2006 Conference, AIAA 2006-7506, pp. 2788-2809, Reston, Va, USA, September 2006.

[6] C. Mathieu and A. L. Weigel, "Assessing the flexibility provided by fractionated spacecraft," in Proceedings of the Space 2005 Conference, AIAA 2005-6700, Long Beach, Calif, USA, AugustSeptember 2005.

[7] M. G. O'Neill, Assessing the impacts of fractionation on pointingintensive spacecraft [M.S. thesis], Institute of Technology, 2010.

[8] D. A. O'Neil and J. C. Mankins, "The advanced technology lifecycle analysis system (ATLAS)," in Proceedings of the 55th International Astronautical Congress, IAC-04-IAA.3.6.3.01, Vancouver, Canada, October 2004.

[9] O. Brown, A. Long, N. Shah, and P. Eremenko, "System lifecycle cost under uncertainty as a design metric encompassing the value of architectural flexibility," in Proceedings of the AIAA Space 2007 Conference, AIAA 2007-6023, pp. 216-229, Long Beach, Calif, USA, September 2007.

[10] J. M. Lafleur and J. H. Saleh, "GT-FAST: a point design tool for rapid fractionated spacecraft sizing and synthesis," in Proceedings of the AIAA Space 2009 Conference and Exposition, AIAA 2009-6563, Pasadena, Calif, USA, September 2009.

[11] J. M. Lafleur and J. H. Saleh, "Exploring the F6 fractionated spacecraft trade space with GT-FAST," in Proceedings of the AIAA Space 2009 Conference and Exposition, AIAA 2009-6802, Pasadena, Calif, USA, September 2009.

[12] W. Yao, X. Q. Chen, and Y. Zhao, "Based on the uncertainty of MDO satellite overall optimization design," Chinese Journal of Aeronautics, vol. 30, no. 5, pp. 68-76, 2009.

[13] W. Yao, X. Q. Chen, W. C. Luo, M. von Tooren, and J. Guo, "Review of uncertainty-based multidisciplinary design optimization methods for aerospace vehicles," Progress in Aerospace Sciences, vol. 47, pp. 450-479, 2011.

[14] M. Daniels, J. Irvine, B. Tracey, W. Schram, and M. E. PatéCornell, "Probabilistic simulation of multi-stage decisions for operation of a fractionated satellite mission," in Proceedings of the IEEE Aerospace Conference (AERO '11), pp. 1-16, March 2011.

[15] P. Collopy, "Value of the probability of success," in Proceedings of the AIAA Space 2008 Conference and Exposition, AIAA 20087868, San Diego, Calif, USA, September 2008. 
[16] S. J. Julier and J. K. Uhlmann, "Unscented filtering and nonlinear estimation," Proceedings of the IEEE, vol. 92, no. 3, pp. 401422, 2004.

[17] R. van der Merwe, E. A. Wan, and S. I. Julier, "Sigma-point kalman filters for nonlinear estimation and sensor-fusion: applications to integrated navigation," in Proceedings of the AIAA Guidance, Navigation, and Control Conference, AIAA 2004-5120, pp. 1735-1764, Providence, RI, USA, August 2004.

[18] P. D. Collopy, "Economic-based distributed optimal design," in Proceedings of the AIAA Space 2001 Conference and Exposition, AIAA 2001-4675, Albuquerque, NM, USA, 2001.

[19] NASA, NASA Systems Engineering Handbook, SP-610S, NASA, 1995.

[20] E. M. Mahr, Small Satellite Cost Model 2007 (SSCM07) User's Manual, ATR-2007(8617)-5, The Aerospace Corporation, 2007.

[21] A. Braukhane, "Lessons learned from one-week concurrent engineering study approach," in Proceedings of the 17th International Conference on Concurrent Enterprising (ICE '01), pp. 1-10, Bremen, Germany, 2011.

[22] R. Hassan and W. Crossley, "Spacecraft reliability-based design optimization under uncertainty including discrete variables," Journal of Spacecraft and Rockets, vol. 45, no. 2, pp. 394-405, 2008. 


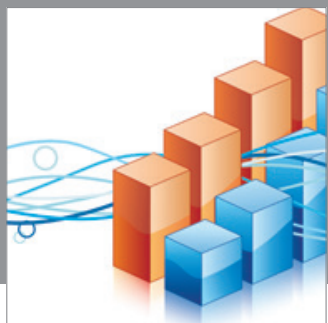

Advances in

Operations Research

mansans

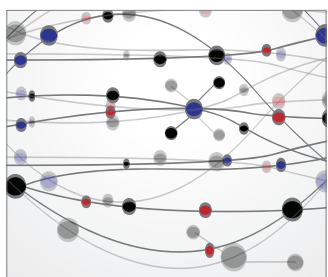

The Scientific World Journal

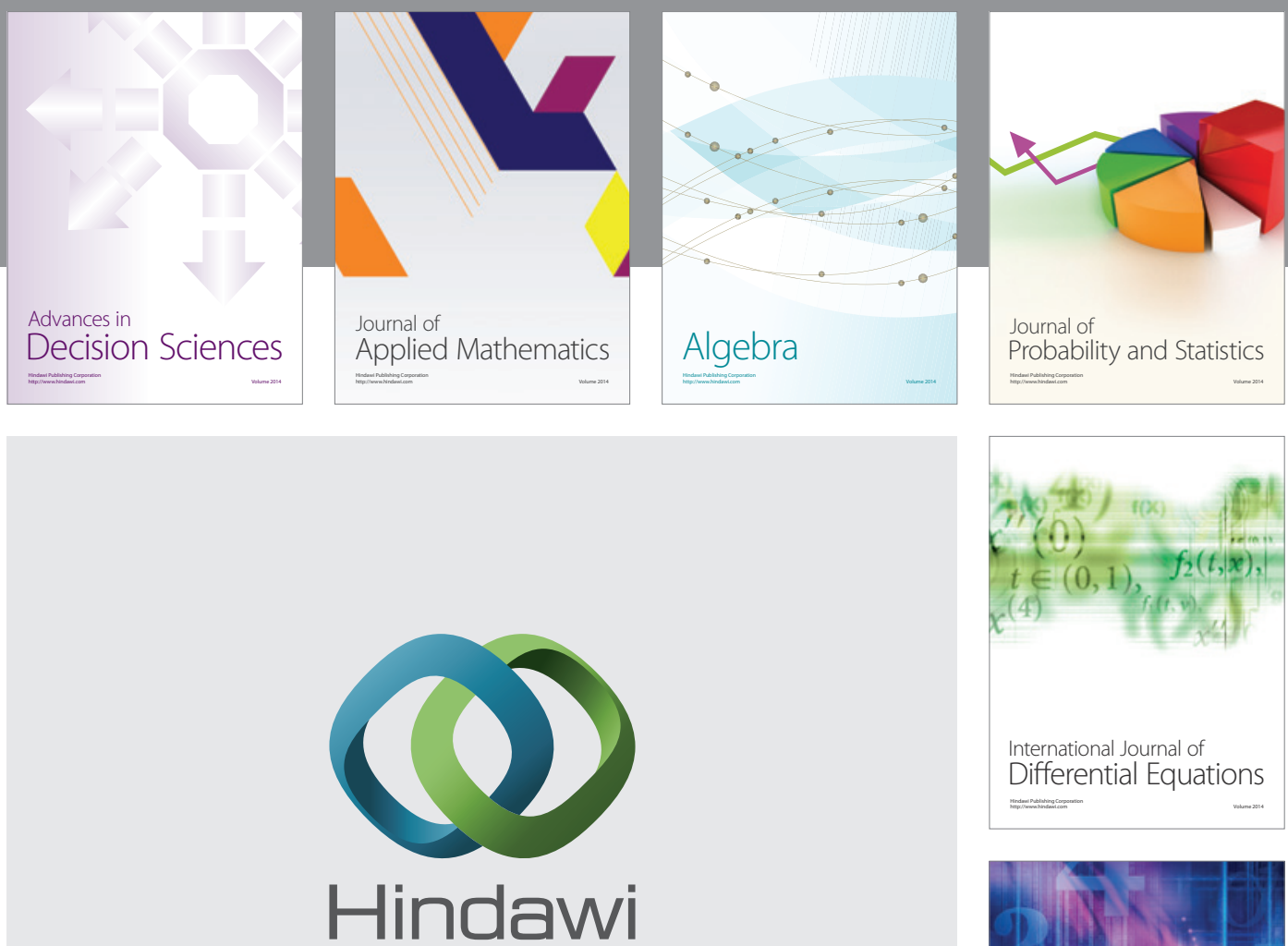

Submit your manuscripts at http://www.hindawi.com
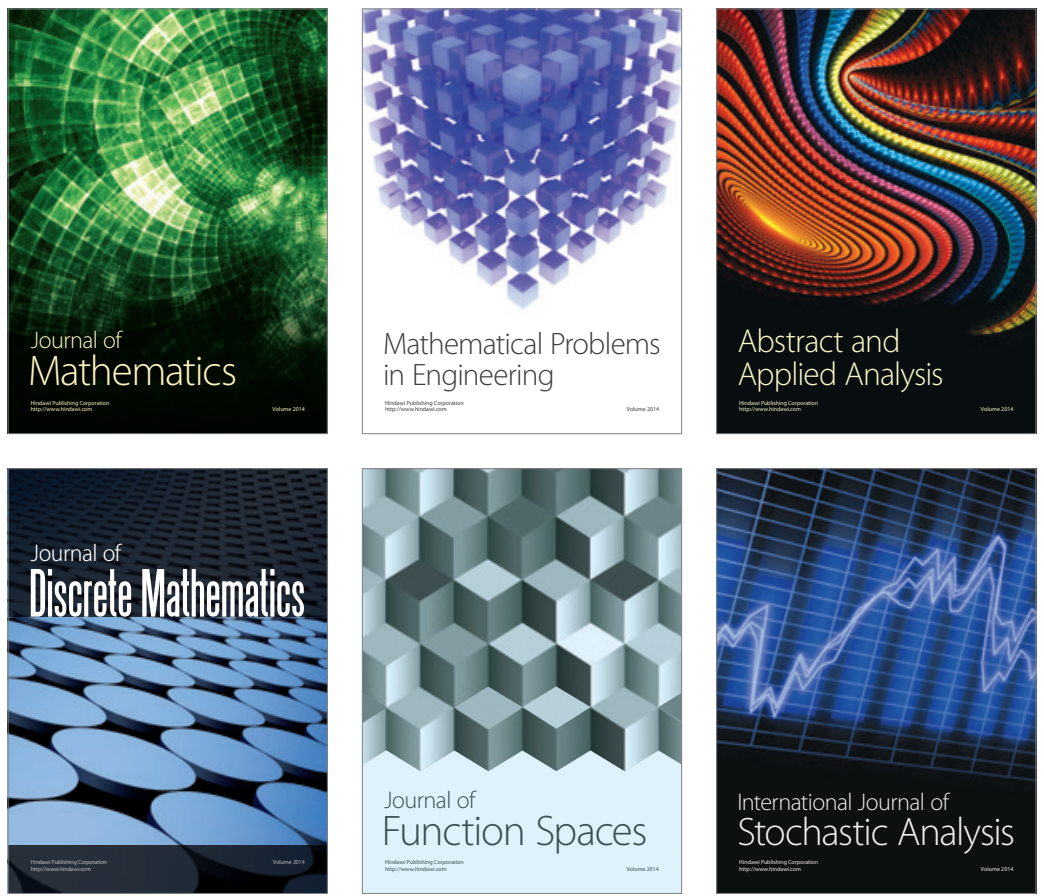

Journal of

Function Spaces

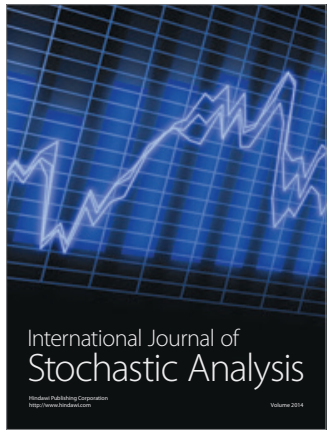

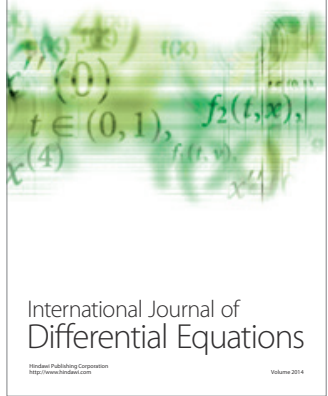
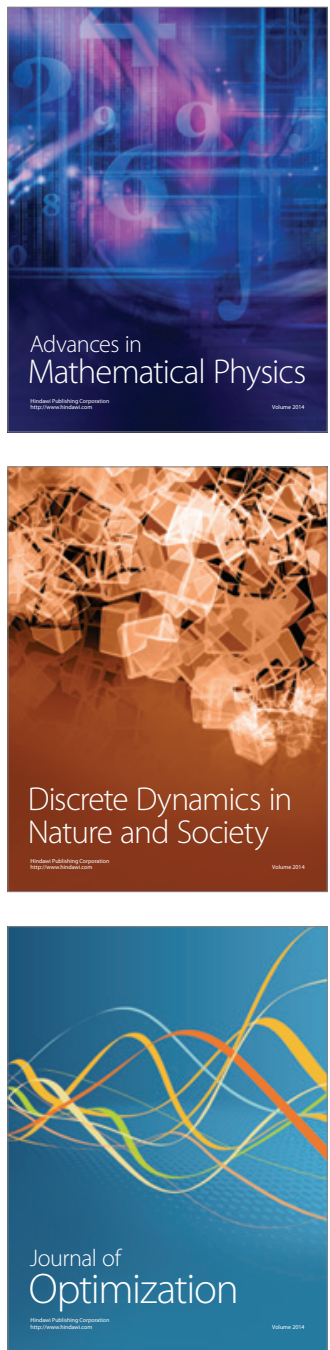